\title{
The Incidence of Early Recurrence After Anatomical Versus Non-Anatomical Liver Resection in Patients with Hepatocellular Carcinoma
}

\author{
Mohamed Abdallah Hablus, Osama Hasan Abd-Raboh \\ General Surgery Department, Faculty of Medicine, Tanta University Hospitals, Tanta, Egypt \\ Email address: \\ dr_osama_hassan@med.tanta.edu.eg (O.H. Abd-Raboh) \\ To cite this article: \\ Mohamed Abdallah Hablus, Osama Hasan Abd-Raboh. The Incidence of Early Recurrence After Anatomical Versus Non-Anatomical Liver \\ Resection in Patients with Hepatocellular Carcinoma. Advances in Surgical Sciences. Vol. 6, No. 1, 2018, pp. 7-15. \\ doi: $10.11648 /$ j.ass. 20180601.12
}

Received: January 17, 2018; Accepted: January 26, 2018; Published: February 19, 2018

\begin{abstract}
Aim: This retrospective study compares the incidence of early recurrence after anatomical versus non-anatomical resection in patients with hepatocellular carcinoma (HCC). Patients and Methods: This retrospective study included 26 patients who had a preoperative diagnosis of a single hepatocellular carcinoma (HCC) and who underwent anatomical and nonanatomical liver resection. The patients were divided into two groups. AR Group; anatomical resection group ( $\mathrm{n}=12$ ) and NAR Group; Non-anatomical resection group $(n=14)$. The effect of the type of resection (anatomic vs non-anatomic) on early HCC recurrence was studied in both groups. Other risk factors that might play a role in early tumor recurrence such as the TNM staging, tumor size, vascular invasion, pathologic grading and high AFP values were also evaluated. Results: During the follow up period, 5 patients (41.7\%) from AR Group and 6 patients (42.9\%) from NAR Group developed recurrence. Mean time of recurrence was $13.05 \pm 4.63$ versus $12.53 \pm 4.12$ months ( $p$ 0.764). The univariate analysis method was used to analyze different epidemiological, clinical and pathological variables and there was no statistically significant risk factor in both groups in terms of recurrence. The mean disease-free survival was calculated for both groups using Kaplan-Meier curve and it was $16.013 \pm 2.324$ for AR group versus $15.657 \pm 2.765$ months for NAR group (p: 0.98). The cumulative overall survival proportion at end of research was $59.6 \%$ for AR group versus $69.87 \%$ for NAR group. The mean overall survival was $17.956 \pm 2.254$ months for AR group versus $20.876 \pm 1.263$ for NAR group (p: 0.21 ). The recurrence rate was $41.7 \% \%$ in AR group and $42.9 \%$ in NAR group $(\mathrm{p}=0.951)$. This suggests that the type of resection did not have an impact on early recurrence in HCC patients undergoing liver resection. Conclusion: This study concluded that recurrence-free and overall survivals after both anatomical and non-anatomical resection were not significantly different from the statistical point of view. Liver resection in cirrhotic patients should be performed with good expertise and should pursue strict selection criteria. Non-Anatomical resection may be more appropriate in cirrhotic patients with small HCC to preserve adequate functioning hepatic parenchyma and to avoid increased postoperative morbidity and mortality.
\end{abstract}

Keywords: HCC, Liver Resection, Local Recurrence, Anatomical Resection, Non-Anatomical Resection

\section{Introduction}

Hepatocellular carcinoma (HCC) is considered one of the most prevalent malignant tumour all around the world [1], and its incidence continues to increase due to various risk factors, particularly hepatitis induced cirrhosis and nonalcoholic steatohepatitis (NASH) [2-4]. additionally, HCC is the 3rd largest cause of cancer related deaths, of 500,000 deaths globally every year [5]. As with most solid tumours, curative liver resection is widely considered the first line therapy for HCC due to its acceptable mortality, morbidity and long term outcomes [6, 7]. Non-anatomic resection (NAR) is focused on achieving a non-tumoural liver parenchyma cuff, without taking into account the Glisson's portal pedicles $[8,9]$. Due to the underlying liver diseases of most patients with hepatocellular carcinoma, such as chronic hepatitis and cirrhosis, NAR is regarded to be beneficial for preserving as much functioning liver parenchyma as possible 
[10]. This technique is extremely crucial in cirrhotic patients because the cirrhotic liver has a very limited ability to regenerate [11], which is closely associated with the longterm outcome. However, a tumour-free margin is not the only factor to be considered regarding HCC recurrence, Kang et al [12] debated that the safety margin width could not offer a better local control rate and that tumour cells could easily spread via the portal venous system rather than only by adjacent diffusion. Anatomical liver resection (AR) is defined as resection of the tumour together with the portal pedicle related to it [13]. Theoretically, AR is able to avoid intrahepatic metastasis and local recurrence due to the invasion of tumour cells along portal veins and their intrasegmental branches $[14,15]$, and it is recommended as a feasible, effective and safe procedure for HCC [16]. However, AR needs to sacrifice a large portion of liver parenchyma to guarantee eradication of potential vascular invasions and daughter nodules and is therefore significantly unfavorable for treating patients with an underlying disease [17]. Furthermore, AR requires complex surgical procedures and highly accurate real-time sonographic guidance. Regarding to long-term survival and recurrence-free survival, no clear evidence is available regarding the superiority of AR compared with NAR.

\section{Aim}

The ideal therapy for HCC remains undetermined due to the contradictory results of the published articles. The present retrospective study compared the incidence of early recurrence after anatomical versus non anatomical resection in patients with hepatocellular carcinoma.

\section{Patients and Methods}

This was a retrospective study which included 26 patients who had a preoperative diagnosis of a single $\mathrm{HCC}$ and who underwent anatomical and nonanatomical liver resection at Surgery Department, Tanta University Hospital Between June 2012 and May 2016, The pre-operative investigations included blood chemistry, hepatitis B \& C markers, alphafetoprotein (AFP), abdominal ultrasonography (US), computed tomography (CT), chest radiography with or without liver biopsy based on the diagnostic criteria of the American Association for the Study of Liver Diseases (AASLD) [19]. All HCC patients were discussed in the multidisciplinary HCC board at Tanta University Hospital. All selected HCC patients for liver resection was Child-Pugh class A with no manifestations of clinically significant portal hypertension (including platelet count of less than 100000/cubic mm, splenomegaly and oesophogeal varices). Additionally, all selected patients were free of any extrahepatic metastasis. The primary outcomes were intrahepatic and local recurrence. Regarding the time of recurrence, early recurrence was defined as recurrence within 2 years after the initial liver resection.

\subsection{Patients Characteristics}

The following clinical variables were compared in the two groups: age, sex, viral markers, presence or absence of cirrhosis, serum albumin, serum total bilirubin, platelet count and serum AFP (Table 1). The patients were divided into two groups: AR Group; Anatomic resection group ( $\mathrm{n}=$ 12) was defined as the complete removal of at least 1 Couinaud's segment containing the tumor together with the related portal vein and the corresponding hepatic arterial branch (Figure 1,2). The appropriate segment margins were identified by the line of demarcation after discoloration of the parenchyma after ligation of the corresponding arterial and portal venous branches and with the help of intraoperative US. NAR Group; Non-anatomic resection group $(\mathrm{n}=14)$ was defined as the resection of the tumor with a safety margin of at least $1 \mathrm{~cm}$ without any consideration to segmental, sectional or lobar anatomy (Figure 3, 4, 5). All patients underwent intraoperative hepatic ultrasonography and were deemed to have resectable tumors at the time of surgery.

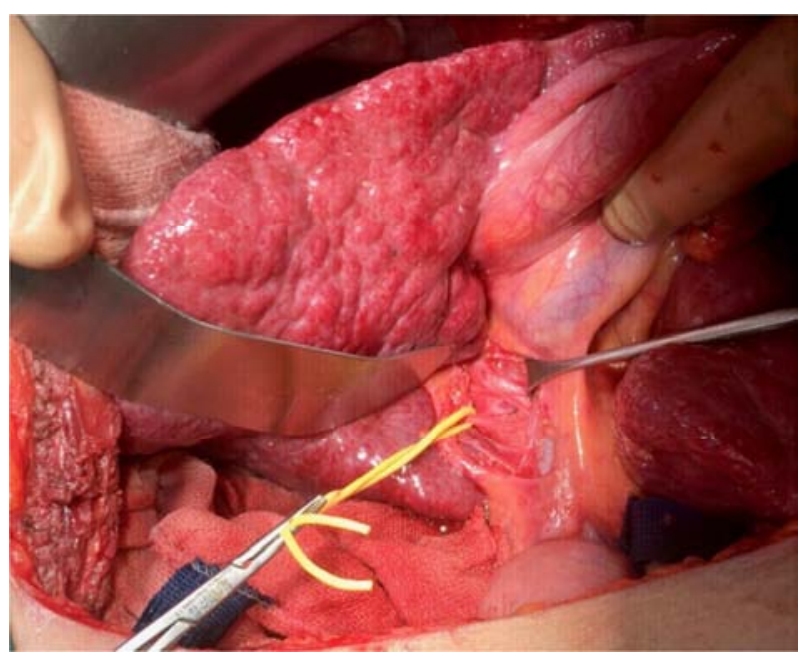

Figure 1. Encircling the right portal vein before its ligation.

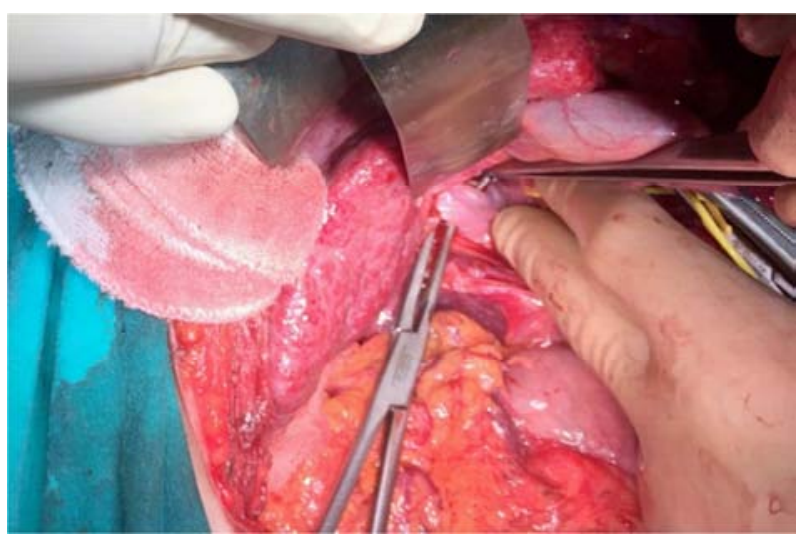

Figure 2. Encircling of the right hepatic artery. 


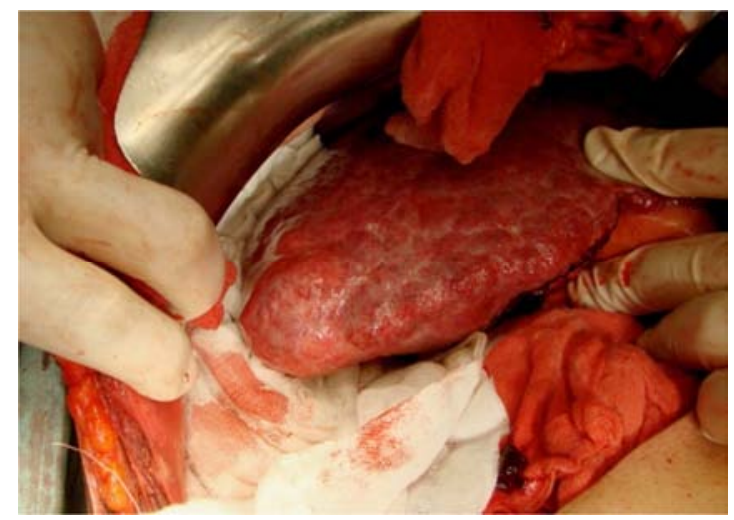

Figure 3. HCC in right lobe (segment VI).

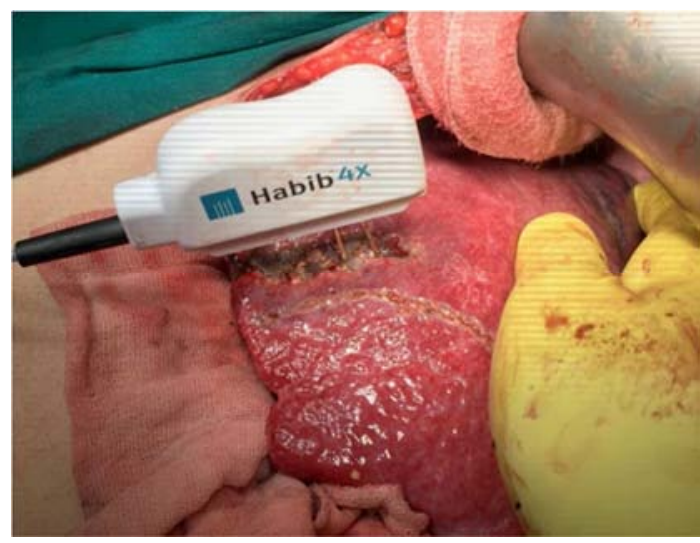

Figure 4. Bipolar radiofrequency device used in cirrhotic liver for non anatomic resection.

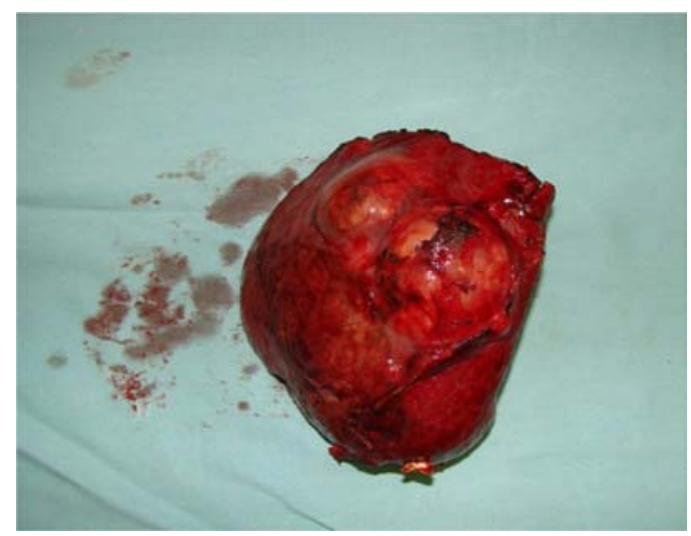

Figure 5. HCC after non anatomic resection.

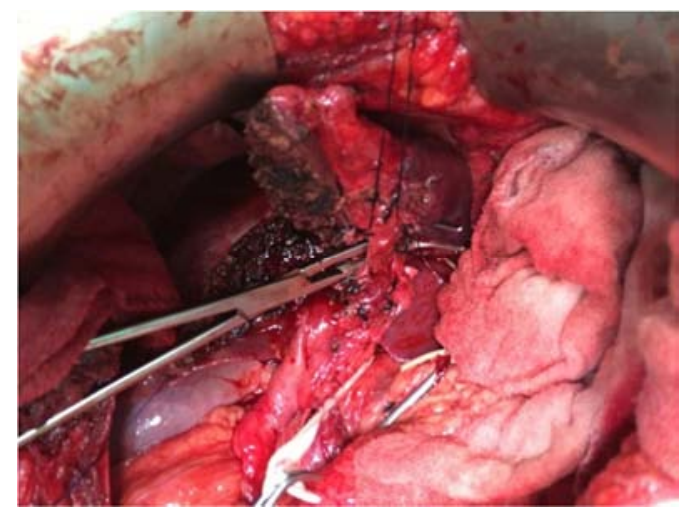

Figure 6. Segment 2 pedicle ligation during left lateral segmentectomy.

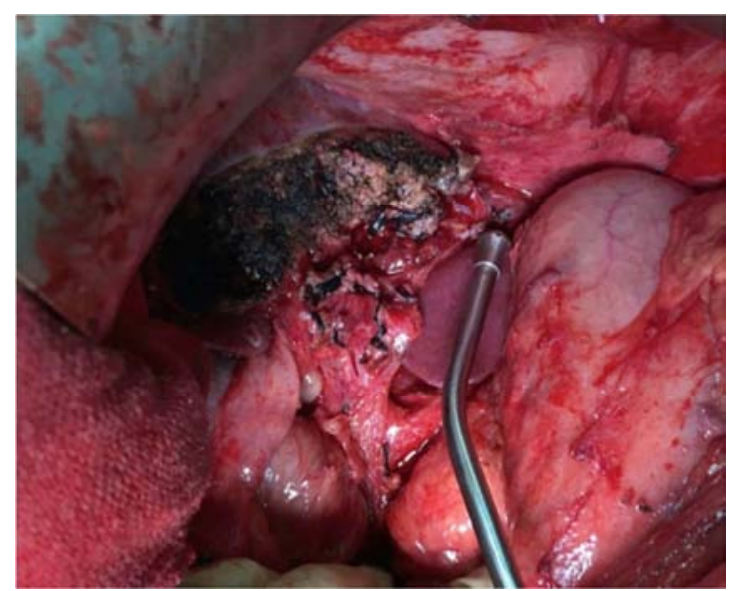

Figure 7. Cut surface after left lateral segmentectomy.

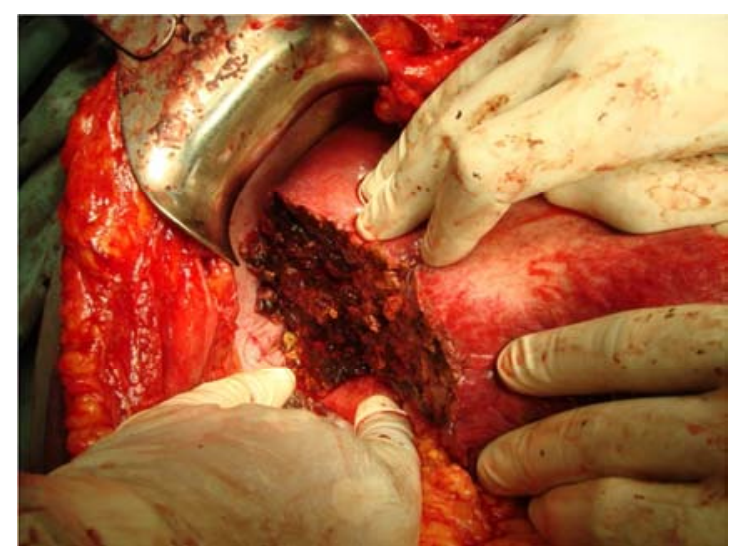

Figure 8. The resection surface after right posterior sectionectomy (Bisegmentectomy VI and VII).

\subsection{Patients Follow-up}

The two groups of patients were subjected to a close follow up for 2 years. During this period of time, they underwent clinical, radiologic (abdominal US and triphasic abdominal CT scan) and biologic (serum AFP and liver function tests) assessment. This evaluation was repeated every 3 months and the patients were seen and discussed in the multidisciplinary HCC board of Tanta University Hospital throughout the follow-up period. The effect of the type of resection (anatomic vs non-anatomic) on early HCC recurrence was studied in both groups. Other risk factors that might play a role in early tumor recurrence such as the TNM staging, tumor size, vascular invasion, pathologic grading and high AFP values were also evaluated.

\subsection{Statistical Analysis}

For continuous variables, data are presented as mean +/Standard Deviation (SD). Group comparisons were performed using univariate analysis (Cox regression test). For multivariate analysis, different factors were correlated with early tumor recurrence (24 months), Survival was calculated using the Kaplan-Meier method (Deltagraph 4.0). $\mathrm{P}$-value $<0.05$ was considered significant. 


\section{Results}

In the present study, there was no statistically significant difference between the 2 groups in terms of clinical and demographic characteristics with respect to age, sex, viral hepatitis markers, the presence of underlying liver cirrhosis, serum albumin, serum bilirubin and AFP levels (Table 1).

Table 1. Pre-operative data.

\begin{tabular}{llll}
\hline Variable & Anatomical $(\mathbf{n = 1 2})$ & Non Anatomical $(\mathbf{n}=\mathbf{1 4})$ & P value \\
\hline Age (years) & $52.31 \pm 5.64$ & $56.36 \pm 6.18$ & 0.096 \\
Sex (M/F) & $9 / 3$ & $8 / 6$ & 0.340 \\
HBV (yes/no) & $4 / 8$ & $4 / 10$ & 0.793 \\
HCV (yes/no) & $9 / 3$ & $10 / 4$ & 0.838 \\
Cirrhosis (yes/no) & $10 / 2$ & $11 / 3$ & 0.759 \\
AFP level (ng/mL) & $112.65 \pm 110.85$ & $135.62 \pm 124.39$ & 0.626 \\
\hline
\end{tabular}

Left hepatectomy was done in 1 case, right hepatectomy was done in 1 case and left lateral segmentectomy in 5 cases (Figure 6, 7), right posterior sectionectomy in one case (Figure 8) while other segmental resections were done in 4 cases, segment III (SIII) resection in 3 cases and segment VI (SVI) resection in one case. Non anatomical resection were done in 14 cases (Table 2).

The mean operative time for AR Group $3.72 \pm 1.18$ versus $3.21 \pm 0.75$ hours for NAR Group. The mean operative blood loss during surgery was $920.52 \pm 325.65$ for AR Group versus $784.36 \pm 195.87$ for NAR Group (Table 3).

Additionally, there was no statistically significant difference between both groups as regards tumor staging (Table 4). Vascular invasion (T3) occurred in 3 patients in the AR Group (25\%) and 4 patients in NAR Group (28.6\%). Nodal involvement was detected in 2 patients in AR group $(16.7 \%)$ and 3 patients in NAR group (21.4\%). Tumour size ranged from 2 to $5 \mathrm{~cm}$ with a mean size of $4.01 \pm 0.85 \mathrm{~cm}$ in AR Group and $3.73 \pm 1.05 \mathrm{~cm}$ in NAR Group (p: 0.647). Tumors were capsulated in 9 patients $(75 \% \%)$ in AR Group and in 10 patients $(71.4 \%)$ in NAR Group. Resection margin was free in all patients of AR Group (100\%) and 13 patients of $(92.9 \%)$ NAR Group patients. Microscopic invasion was found in $3(25 \%)$ of AR Group patients and in 4 of $(28.6 \%)$
NAR Group patients. (Table 5) shows the pathological types and differentiation of tumors in the studied groups of patients. Tumours were mostly well differentiate pure HCC in most of the patients. One patient had fibrolamellar HCC and another one had mixed HCC and Cholangiocarcinoma.

Diaphragm involvement requiring resection occurred in a single patient in AR group (8.3\%). One patient of NAR group had haemorrhage due to injury of one of the short hepatic veins during mobilization of the right hemiliver of the inferior vena cava and it was controlled by suturing with Prolene 3/0. As regards postoperative complications (table 6), there was no statistically significant difference between both groups $(16.6 \%$ vs $21.4 \%)$. There were early post operative complications in 7 patients $(58.3 \%)$ in AR group and 3 patients $(21.4 \%)$ in NAR group. The most frequent complication was intraabdominal collection, where ultrasonography guided tube drainage was done. Postoperative atelectasis and mild pleural effusion were treated medically, while chest tube was inserted in one case with bilateral pleural effusion. Biliary leakage was minor and it was managed conservatively within 11 days. Hospital stay (table 6) was not significantly different between the anatomic and non-anatomic groups $(9.34 \pm 4.56$ vs $7.35 \pm 2.69)$ with a p-value of 0.181 .

Table 2. Types of hepatic resection in the studied groups.

\begin{tabular}{|c|c|c|c|c|}
\hline & \multicolumn{2}{|l|}{ Anatomical resection group } & \multicolumn{2}{|c|}{ Non Anatomical resection group } \\
\hline No. & 12 & & 14 & \\
\hline \multirow{4}{*}{ Type } & Left hepatectomy & $1(8.3 \%)$ & III & $4(28.6 \%)$ \\
\hline & Left lateral & $5(41.7 \%)$ & IV b & $2(14.3 \%)$ \\
\hline & Segmental: SIII in 3 caes, SVI in 1 case and bisegmentectomy VI \& VII in one case & $5(41.7 \%)$ & $\mathrm{V}$ & $2(14.3 \%)$ \\
\hline & Right hepatectomy & $1(8.3 \%)$ & VI & $6(42.9 \%)$ \\
\hline
\end{tabular}

Table 3. Comparison of operative data between both groups.

\begin{tabular}{lllll}
\hline & & Anatomical $(\mathbf{n}=12)$ & Non Anatomical $(\mathbf{n}=\mathbf{1 4})$ & P value \\
\hline \multirow{2}{*}{ Operative time (hours) } & Range & $2.9-5.1$ & $2.4-3.8$ & 0.194 \\
\multirow{2}{*}{ Blood loss (ml) } & Mean \pm SD & $3.72 \pm 1.18$ & $3.21 \pm 0.75$ & \\
& Range & $250-1500$ & $500-100$ & 0.201 \\
\hline
\end{tabular}

Table 4. Histopathological data of both groups.

\begin{tabular}{lllll}
\hline & & Anatomical $(\mathbf{n}=12)$ & Non Anatomical (n=14) & P value \\
\hline \multirow{2}{*}{ Liver } & Cirrhotic (\%) & $10(83.3 \%)$ & $11(78.6 \%)$ & 0.759 \\
Mean Tumor size $(\mathrm{cm})$ & Non cirrhotic (\%) & $2(16.7 \%)$ & $3(21.4 \%)$ & 0.647 \\
\multirow{2}{*}{ Tumor capsule } & & $4.01 \pm 0.85$ & $3.73 \pm 1.05$ & $10(71.4 \%)$ \\
\hline
\end{tabular}




\begin{tabular}{lllll}
\hline & & Anatomical $(\mathbf{n}=12)$ & Non Anatomical $(\mathbf{n}=14)$ & P value \\
\hline \multirow{2}{*}{ Resection margin } & Free (\%) & $12(100 \%)$ & $13(92.9 \%)$ & 0.345 \\
\multirow{2}{*}{ Microscopic invasion } & Infiltrated (\%) & $0(0 \%)$ & $1(7.1 \%)$ & \\
& Yes (\%) & $3(25 \%)$ & $4(28.6 \%)$ & 0.838 \\
\multirow{2}{*}{ Nodal involvement } & No (\%) & $9(75 \%)$ & $10(71.4 \%)$ & \multirow{2}{*}{0.759} \\
\hline
\end{tabular}

Table 5. Pathological types and differentiation of tumors in the studied groups.

\begin{tabular}{llll}
\hline & Anatomical $(\mathbf{n}=\mathbf{1 2})$ & Non Anatomical $(\mathbf{n}=\mathbf{1 4})$ & P value \\
\hline HCC: & $11(91.7 \%)$ & $13(92.9 \%)$ & 0.910 \\
$\quad$ Well differentiated (grade I) & $7(58.3 \%)$ & $6(42.9 \%)$ & \\
$\quad$ Moderately differentiated (grade II) & $2(16.7 \%)$ & $4(28.6 \%)$ & \\
$\quad$ Poorly differentiated (grade III) & $2(16.7 \%)$ & $3(21.4 \%)$ & 0.271 \\
Fibrolamellar HCC & $1(8.3 \%)$ & $0(0 \%)$ & 0.345 \\
Mixed HCC-CC & $0(0 \%)$ & $1(7.1 \%)$ & \\
\hline
\end{tabular}

Table 6. Postoperative data of both studied groups.

\begin{tabular}{|c|c|c|c|c|}
\hline & & Anatomical (n=12) & Non Anatomical $(n=14)$ & P value \\
\hline Hospital stay (days) & $\begin{array}{l}\text { Range } \\
\text { Mean } \pm \text { SD }\end{array}$ & $\begin{array}{l}5-28 \\
9.34 \pm 4.56\end{array}$ & $\begin{array}{l}4-16 \\
7.35 \pm 2.69\end{array}$ & 0.181 \\
\hline $\begin{array}{l}\text { Postoperative complications } \\
\text { Late complications }\end{array}$ & & $\begin{array}{l}7(58.3 \%) \\
1(8.3 \%)\end{array}$ & $\begin{array}{l}3(21.4 \%) \\
1(7.1 \%)\end{array}$ & $\begin{array}{l}0.054 \\
0.910\end{array}$ \\
\hline
\end{tabular}

The perioperative mortality rates (table 7) were not statistically significant between both groups $(\mathrm{p}=0.653)$. There were four perioperative mortality cases in AR group and 3 cases in NAR group. In AR group, severe deterioration in liver functions and eventually death occurred in 2 patients, pulmonary embolism in one patient and postoperative haemorrhagic shock in other patient. While in NAR group there were three perioperative mortality cases, post hepatectomy liver cell failure in 2 patients and adult respiratory distress syndrome (ARDS) leading to death in 1 patient. Two patients were found to have incisional hernia during the period of follow up one in each group.

During the follow up period, 5 patients $(41.7 \%)$ from AR Group and 6 patients (42.9\%) from NAR Group developed recurrence (table 7). Mean time of recurrence was $13.05 \pm$ 4.63 versus $12.53 \pm 4.12$ months ( $\mathrm{p} 0.764$ ). Two patients from each group developed distant metastasis including brain, lung and supraclavicular lymph node. TACE percutaneous radiofrequency ablation or supportive medical management were given according to general condition and liver function.

The univariate analysis method (table 8) was used to analyze different epidemiological, clinical and pathological variables and there was no statistically significant risk factor in both groups in terms of recurrence. The recurrence rate was $41.7 \% \%$ in AR group and $42.9 \%$ in NAR group ( $p=$ 0.951). This suggests that the type of resection did not have an impact on early recurrence in HCC patients undergoing liver resection.

Table 7. Recurrence and mortality comparison between both groups.

\begin{tabular}{llll}
\hline & Anatomical (n=12) & Non Anatomical (n=14) & P value \\
\hline Early recurrence within 2 years (\%) & $5(41.7 \%)$ & $6(42.9 \%)$ & 0.951 \\
Mean time of recurrence (months) & $13.05 \pm 4.63$ & $12.53 \pm 4.12$ & 0.764 \\
Mortality (\%) & $4(33.3 \%)$ & $3(21.4 \%)$ & 0.653 \\
\hline
\end{tabular}

Table 8. Univariate analysis of factors affecting Disease Free Survival (DFS).

\begin{tabular}{lllll}
\hline & & $\begin{array}{l}\text { Anatomical resection group: mean survival } \\
\text { (months) }\end{array}$ & $\begin{array}{l}\text { Non-Anatomical resection group: mean survival } \\
\text { (months) }\end{array}$ \\
\hline \multirow{2}{*}{ Age } & $\leq 60$ years & $18.7 \pm 5.64$ & $22.4 \pm 4.53$ \\
& $>60$ years & $15.8 \pm 4.12 \#$ & $21.12 \pm 5.31$ \\
Sex & Male & $17.9 \pm 6.4$ & $21.4 \pm 4.85$ \\
& Female & $20.4 \pm 5.9$ & $20.8 \pm 5.1$ & 0.124 \\
Liver cirrhosis & Cirrhotic & $16.8 \pm 3.9$ & $21.1 \pm 4.2$ & $17.9 \pm 4.2$ \\
& Non-Cirrhotic & $23.3 \pm 4.9$ & $18.96 \pm 3.97$ \\
Capsule & No & $18.75 \pm 4.32$ & $21.8 \pm 4.97$ \\
& Yes & $15.74 \pm 5.14$ & $21.7 \pm 5.39$ \\
Resection margin & Free & $18.52 \pm 0$ & & 0.105 \\
Microscopic & Infiltrated & $14.9 \pm 0 \#$ & $18.3 \pm 4.87$ \\
invasion & Yes & $16.8 \pm 5.17$ & $23.1 \pm 4.18$ \\
\hline
\end{tabular}

\# Calculation of p not possible due to little number of patients. 
The mean disease-free survival (DFS) was calculated for both groups using Kaplan -Meier curve and it was $16.013 \pm 2.324$ for AR group versus $15.657 \pm 2.765$ months for NAR group (p: 0.98). The cumulative overall survival (OAS) proportion at end of research was $59.6 \%$ for AR group versus
$69.87 \%$ for NAR group. The mean overall survival (OAS) was 17.956 \pm 2.254 months for AR group versus $20.876 \pm 1.263$ for NAR group (p: 0.21). (Figure 9) compares the disease-free survival (DFS) and (Figure 10) compares the cumulative overall survival (OAS) between both groups.

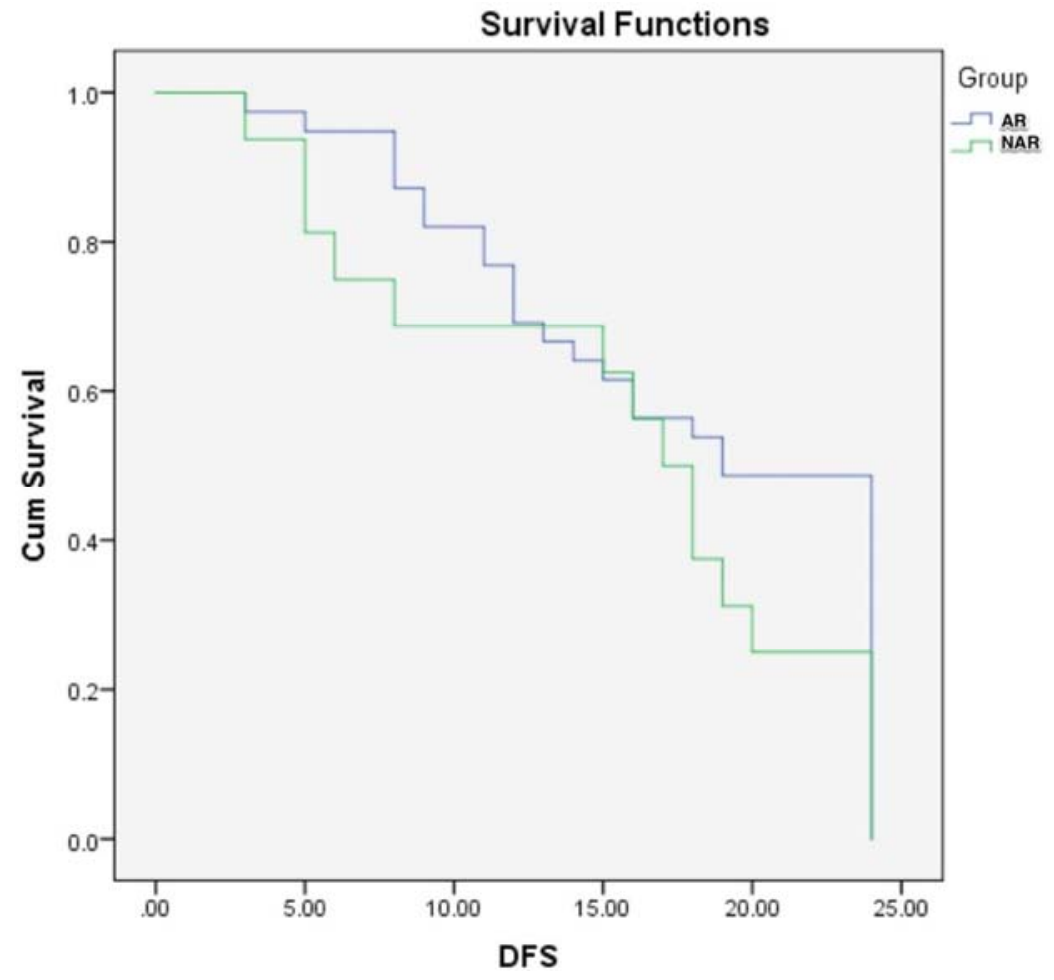

Figure 9. Kaplan-Meier curve compares the disease-free survival (DFS) between both groups (blue line: Anatomical Resection group, green line: NonAnatomical Resection group).

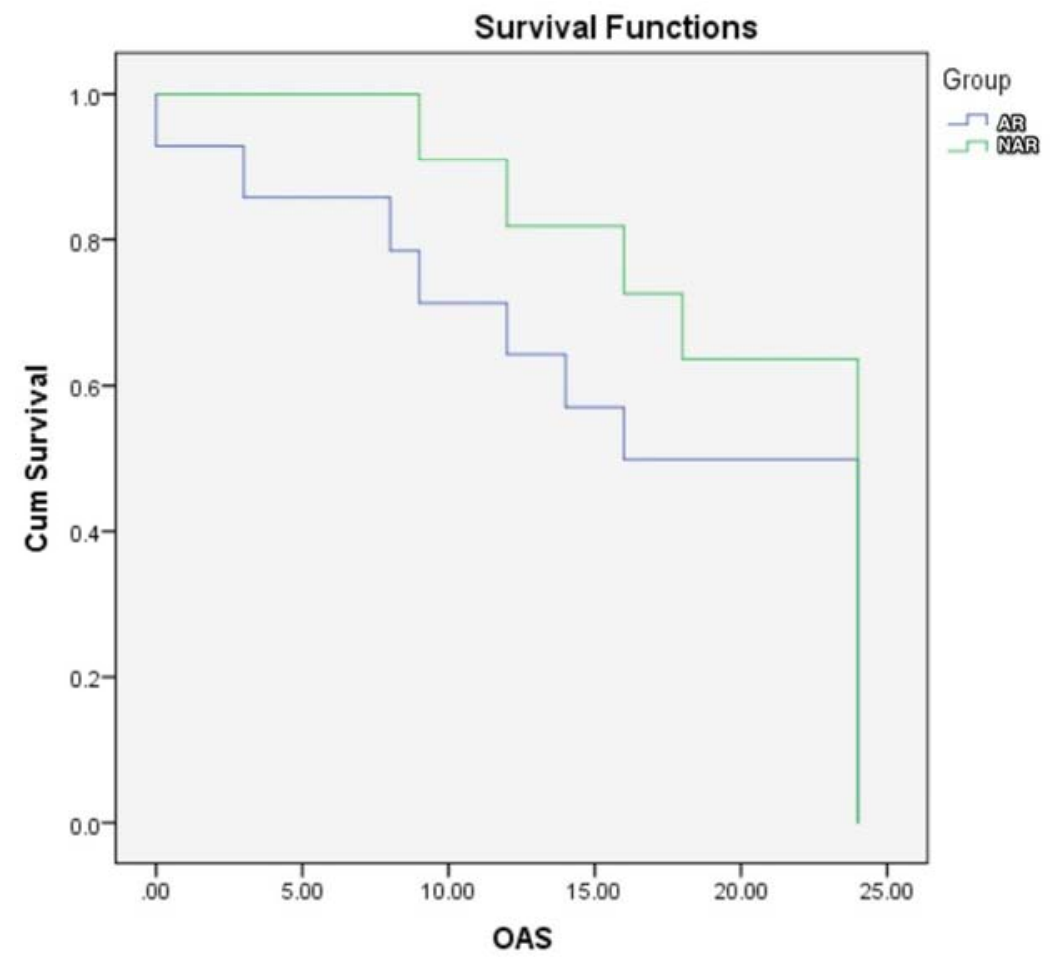

Figure 10. Kaplan-Meier curve compares the cumulative overall survival (OAS) between both groups (blue line: Anatomical Resection group, green line: Non-Anatomical Resection group). 


\section{Discussion}

Several therapeutic options are available for the treatment of patients with hepatocellular carcinoma, and the ideal option is chosen based on the tumour burden and underlying liver disease and most important liver cirrhosis. anatomic or non-anatomic resection and liver transplantation, are the main therapeutic options for HCC patients. The tumor size \& site, the presence of underlying liver cirrhosis and the surgeon's experience are the main factors based on which the type of resection was decided [20]. Both AR and NAR have their own advantages and limitations. AR removes both the neoplasm and potential pre-cancerous hepatic parenchyma, but with an increased risk of post hepatectomy liver failure, which occurs less commonly in HCC patients after NAR [18 \& 20]. Whether AR is superior to NAR or not remains undetermined due to the heterogeneous groups of patients enrolled in previous studies, in which patients had different tumour burdens and underlying liver disease [21].

The present retrospective study tried to assess the effect of the type of liver resection (anatomical versus nonanatomical) in a homogenous group of patients with solitary $\mathrm{HCC}$ in early cirrhotic liver. The patients were similar in preoperative clinical characteristics and tumor biology. This study revealed that the type of resection is not considered a risk factor for early tumor recurrence. While some studies and meta-analysis have found that anatomic resection have a beneficial impact on recurrence-free survival for HCC [19], others have found that anatomic and non-anatomic resection had no significant effect on the risk of HCC recurrence [2224]. These studies were based on long-term overall and disease free survival and therefore early and late recurrence risk factors were not taken into account.

In this study all patients undergoing non-anatomic resection had a $1 \mathrm{~cm}$ clear margin using bipolar radiofrequency device in cirrhotic patients (Habib Tm $4 \mathrm{x}$ generator $1500 \times$ RITA medical systems Inc. California) (Figure 4). A study conducted by Cucchetti et al [25] who compared different risk factors for early and late recurrence in HCC patients with cirrhotic liver. They concluded that the type of liver resection (anatomical versus non-anatomical) is not considered a risk factor for early tumor recurrence which goes in accordance with the results of this study. Some Asian and European studies have reported the survival benefit of anatomical or segmental liver resection in HCC patients [2630]. From an oncological point of view, anatomical liver resection may be ideal, because malignant cells are thought to spread along locoregional Glisson's portal pedicle in the same segment as other intrahepatic tumours [31]. Regimbeau et al. [23] reported shorter recurrence-free and overall survival rates and a higher intrahepatic recurrence rate in HCC patients who were subjected to non anatomical resection compared to anatomic resection. Mazziotti et al. [27] reported the beneficial effect of anatomical resection for HCC even in compensated liver cirrhosis patients. However, the advantageous impact of anatomical resection compared to non anatomical hepatic resection has always been controversial, although non anatomical hepatic resection seems to be a better option for a small size or small number of HCC or in extremely decompensated cirrhotic patients [32]. No randomized controlled studies have been conducted to differentiate the impact of anatomical and non-anatomical resection [33] for HCC under the same condition concerning tumor burden and underlying liver disease.

In a study conducted by Seheon Kim et al [34], the anatomical resection group showed better disease free survival (DFS) and overall survival (OS) outcomes than the nonanatomical resection group, although the results of DFS and OS were not statistically significant. Further, their study revealed that the anatomical resection group showed results that are more beneficial in relative risk of OS. They explained that the reason there is no statistical difference may be due to the difference between the follow-up periods of the two groups. In another study by Nanashima et al [35], the local recurrence rate was similar between the non-anatomical resection and anatomical resection groups. Disease-free and overall survival in the anatomical resection and nonanatomical resection groups with a negative surgical margin were not significantly different despite the degree of liver dysfunction and the surgical margin was not related to the outcome. Survival in the non-anatomical resection group with a positive surgical margin was extremely poor. They concluded that when non-anatomic resection was selected, a surgical margin without tumor exposure may provide better survival.

\section{Conclusion}

This retrospective study was conducted on HCC patients who underwent anatomical or non-anatomical liver resection, and analyzed different epidemiological, clinical and pathological background and outcomes. The early recurrence rate was similar for both anatomical and non-anatomical resections. Recurrencefree and overall survivals after both anatomical and nonanatomical resection were not significantly different from the statistical point of view. Liver resection in cirrhotic patients should be performed with good expertise and should pursue strict selection criteria. Non-Anatomical resection may be more appropriate in cirrhotic patients with small HCC to preserve adequate functioning hepatic parenchyma and to avoid increased postoperative morbidity and mortality.

\section{References}

[1] Cucchetti A, Qiao G-L, Cescon M, Li J, Xia Y, Ercolani G, et al. (2014): Anatomic versus nonanatomic resection in cirrhotic patients with early hepatocellular carcinoma. Surgery. 155 (3):512 \pm 21.

[2] Altekruse SF, McGlynn KA, Reichman ME. (2009): Hepatocellular carcinoma incidence, mortality, and survival trends in the United States from 1975 to 2005. Journal of clinical oncology: official journal of the American Society of Clinical Oncology. 27 (9):1485 991. 
[3] Njei B, Rotman Y, Ditah I, Lim JK. (2015): Emerging trends in hepatocellular carcinoma incidence and mortality. Hepatology (Baltimore, Md). 61 (1):191 \pm 9.

[4] Jemal A, Bray F, Center MM, Ferlay J, Ward E, Forman D. (2011): Global cancer statistics. cancer journal for clinicians. $61(2): 69 \pm 90$

[5] Altekruse SF, Henley SJ, Cucinelli JE, McGlynn KA. (2014): Changing hepatocellular carcinoma incidence and liver cancer mortality rates in the United States. The American journal of gastroenterology. 109 (4):542 \pm 53 .

[6] Dahiya D, Wu T-J, Lee C-F, Chan K-M, Lee W-C, Chen M-F. (2010): Minor versus major hepatic resection for small hepatocellular carcinoma (HCC) in cirrhotic patients: a 20year experience. Surgery. 147 (5):676 \pm 85 .

[7] Yamazaki O, Matsuyama M, Horii K, Kanazawa A, Shimizu S, Uenishi T, et al. (2010): Comparison of the outcomes between anatomical resection and limited resection for single hepatocellular carcinomas no larger than $5 \mathrm{~cm}$ in diameter: a single-center study. Journal of hepato-biliary-pancreatic sciences. 17 (3):349 \pm 58 .

[8] Hasegawa K, Kokudo N, Imamura H, Matsuyama Y, Aoki T, Minagawa $M$, et al. (2005): Prognostic impact of anatomic resection for hepatocellular carcinoma. Annals of surgery. 242 (2):252 \pm 9 .

[9] Regimbeau J-M, Kianmanesh $R$, Farges $O$, Dondero $F$, Sauvanet A, Belghiti J. (2002): Extent of liver resection influences the outcome in patients with cirrhosis and small

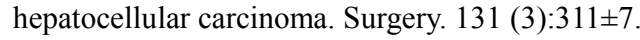

[10] Hsia CY, Lui WY, Chau GY, King KL, Loong CC, Wu CW. (2000): Perioperative safety and prognosis in hepatocellular carcinoma patients with impaired liver function. Journal of the American College of Surgeons. 190 (5):574 \pm 9. PMID: 10801024.

[11] Yamanaka N, Okamoto E, Kawamura E, Kato T, Oriyama T, Fujimoto J, et al. (1993): Dynamics of normal and injured human liver regeneration after hepatectomy as assessed on the basis of computed tomography and liver function. Hepatology (Baltimore, Md). 18 (1):79 \pm 85 .

[12] Kang CM, Choi GH, Kim DH, Choi SB, Kim KS, Choi JS, et al. (2010): Revisiting the role of nonanatomic resection of small $(<$ or $=4 \mathrm{~cm})$ and single hepatocellular carcinoma in patients with well-preserved liver function. The Journal of surgical research. $160(1): 81 \pm 9$.

[13] Popescu I, Campeanu I. (2009): [Surgical anatomy of the liver and liver resection. Brisbane 2000 Terminology]. 104 (1): $7 \pm 10$.

[14] Makuuchi M, Imamura H, Sugawara Y, Takayama T. (2002): Progress in surgical treatment of hepatocellular carcinoma. Oncology. 62 Suppl 1:74 \pm 81 .

[15] Kim YK, Han HS, Yoon YS, Cho JY, Lee W. (2015): Total anatomical laparoscopic liver resection of segment 4 (S4), extended S4, and subsegments S4a and S4b for hepatocellular carcinoma. Journal of laparoendoscopic \& advanced surgical techniques Part A. 25 (5):375 \pm 9.

[16] Thuan N, Long TCD, Bac NH, Dat LT, Viet DQ. (2014): Glissonean pedicle transection for anatomic liver resection. HPB. 2014; 16:466.
[17] Kudo A, Tanaka S, Ban D, Matsumura S, Irie T, Nakamura N, et al. (2014): Anatomic resection reduces the recurrence of solitary hepatocellular carcinoma $<=5 \mathrm{~cm}$ without macrovascular invasion. American journal of surgery. 207

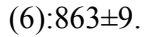

[18] Tomimaru Y, Eguchi H, Marubashi S, Wada H, Kobayashi S, Tanemura M, et al. (2012): Equivalent outcomes after anatomical and non-anatomical resection of small hepatocellular carcinoma in patients with preserved liver function. Digestive diseases and sciences. 57 (7):1942 \pm 8 .

[19] Eltawil KM, Kidd M, Giovinazzo F, Helmy AH and Salem RR (2010): Differentiating the impact of anatomical and non anatomical liver resection on early recurrence in patients with Hepatocellular carcinoma: World J of Surg. Oncology 8:1-7.

[20] Tan Y, Zhaung W, Jiang L, Yang J and Yan L (2017): Efficacy and safety of anatomic versus non anatomic resection in patients with hepatocellular carcinoma: A systemic review and meta analysis. PloS ONE 12 (10):e0186930.

[21] Abdelhady H, Hossam I, Ismaeil T, Elsheikh M, Abdelwahab $M$ Soliman $M$ and Hassan $O$ (2015): Anatomic versus non anatomic Hepatic resection for patients with stage I and II Hepatocellular carcinoma; Ain Shams J Surg 8 (2):151-162.

[22] Torzilli G, Makuuchi M, Inoue K, Takayama T, Sakamoto Y, Sugawara Y, Kubota K and Zucchi A. (1999): No-mortality liver resection for hepatocellular carcinoma in cirrhotic and noncirrhotic patients: is there a way? A prospective analysis of our approach. Arch Surg. 134:984-92.

[23] Regimbeau J. M., Kianmanesh R., Farges O., Dondero F., Sauvanet A. and Belghiti J. (2002): Extent of liver resection influences the outcome in patients with cirrhosis and small hepatocellular carcinoma. Surgery, 131: 311-7.

[24] Ziparo V., Balducci G., Lucandri G., Mercantini P., Giacomo $G$. and Fernandes E. (2002): Indications and results of resection for hepatocellular carcinoma. Eur J Surg Oncol, 28: 723-8.

[25] Cucchetti A, Piscaglia F, Caturelli E, Benvegnu L, Vivarelli M, Ercolani G, Cescon M, Ravaioli M, Grazi GL, Bolondi $L$ and Pinna AD. (2009): Comparison of recurrence of hepatocellular carcinoma after resection in patients with cirrhosis to its occurrence in a surveilled cirrhotic population. Ann Surg Oncol. 16:413-22.

[26] Emond J. C. and Polastri R. (1996): Anatomical hepatectomy for resection or transplantation. Am J Surg, 172: 29-34.

[27] Mazziotti I A., Grazi G. L. and Ercolani G. (1997): Hepatic resective surgery in cirrhotic patients. Techniques and results of anatomical segmentectomies. Ann Ital Chir, 68: 781-90.

[28] Takayama T., Makuuchi M., Yamasaki S., Kosuge T., Yamamoto J. and Shimada K. (1998): Systematic resection for hepatocellular carcinoma. Jpn Surg Soc, 99: 241-4.

[29] Imamura H., Matsuyama Y. and Miyagawa Y. (1999): Prognostic significance of anatomical resection and desgamma-carboxy prothrombin in patients with hepatocellular carcinoma. Br J Surg, 86: 1032-8.

[30] Imamura H., Matsuyama Y. and Tanaka E. (2003): Risk factors contributing to early and late phase intrahepatic recurrence of hepatocellular carcinoma after hepatectomy. J Hepatol, 38: 237-9. 
[31] Shirabe K., Kanematsu T., Matsumata T., Adachi E., Akazawa K. and Sugimachi K. (1991): Factors linked to early recurrence of small hepatocellular carcinoma after hepatectomy: univariate and multivariate analysis. Hepatology, 14: 802-5.

[32] Scheele J. (2001): Anatomical and atypical liver resections. Chirurg. 72: 113-24.

[33] Kanematsu t., Takenaka k., Matsumata t., Furuta t., Sugimachi $k$. and Inokuchi $k$. (1984): Limited hepatic resection effective for selected cirrhotic patients with primary liver cancer. Ann Surg, 199: 51-6.
[34] Seheon K, Seokwhan K, Insaug S, Kwangsik C (2015): Comparison of survival outcomes after anatomical resection and non anatomical resection in patients with Hepatocellular carcinoma. Korean J Hepatobiliary Pancreatic Surg 19:161166.

[35] Nanashima A, Sumida Y, Abo T, Nagasaki T, Tobinaga S, Fukuoka H, Takeshita H, Hidaka S, Tanaka K, Sawai T, Yasutake T and Nagayasu T. (2008): Comparison of survival between anatomic and non-anatomic liver resection in patients with hepatocellular carcinoma: significance of surgical margin in non-anatomic resection. Acta Chir Belg. 108 (5):532-7. 